\title{
A simulação realística como ferramenta de ensino em uma pós-graduação de farmácia clínica: relato de experiência
}

\author{
Realistic simulation as educational tool in a \\ post-graduation of clinical pharmacy: experience report
}

\author{
Marcilene Araújo', Valéria Araújo Barros², Anderson Bentes de Lima \\ Centro Universitário do Pará, Belém (PA), Brasil. \\ ${ }^{2}$ Universidade Federal do Pará Belém (PA), Brasil. \\ ${ }^{3}$ Universidade do Estado do Pará, Belém (PA), Brasil.
}

\section{RESUMO}

Contextualização: A simulação realística compreende uma técnica para substituir ou ampliar experiências reais. Faz parte de uma nova perspectiva de ensino que possibilita não somente as habilidades técnicas, relacionais, éticas e atitudes. Na área da saúde especificamente se apresenta como uma tentativa de reproduzir os aspectos essenciais de um cenário clínico para que, quando um cenário semelhante ocorrer em um contexto clínico real, a situação possa ser gerenciada pela equipe com êxito. O objetivo do presente estudo é apresentar a experiência da simulação realística vivenciada no curso de Pós-graduação em Farmácia Clínica do Centro Universitário do Estado do Pará - CESUPA, como processo de ensino aprendizagem. Descrição da experiência: A pesquisa foi de caráter descritivo e observacional, com abordagem qualitativa. A simulação realística, como ferramenta de ensino- aprendizagem, foi muito bem desenvolvida e aplicada. Preparou os profissionais para a prática clínica, superando as falhas e o medo, contribuindo para a formação com qualidade, representando assim um importante avanço tecnológico no ensino da pós-graduação. Considerações finais: Os discentes conseguiram praticar as habilidades e competências, discutir os casos clínicos simulados e isso somou na construção do conhecimento profissional e no desenvolvimento do raciocínio critico e clínico, tanto na tomada de decisão, na criação de um plano de intervenção, quanto no acompanhamento e na avaliação dos resultados.

Recebido: Jun. 11, 2017

Aceito: Nov. 21, 2017

\section{COMO CITAR ESTE ARTIGO}

Araújo M, Barros VA, Lima AB. A

simulação realística como ferramenta de ensino em uma pós-graduação de farmácia clínica: relato de experiência. Interdisciplinary Journal of Health Interdisciplinary Journal of Health
Education. 2017 Jul-Dez;2(2):115-118. Education. 2017 Jul-Dez;2(2):115-118.
https://doi.org/10.4322/ijhe.2017.007

\section{CORRESPONDÊNCIA}

Anderson Bentes de Lima

Universidade Federal do Pará

Travessa 9 de Janeiro, 1034, São Brás, CEP 66060-575, Belém (PA), Brasil andersonbentes@uepa.br

\section{FONTE DE FINANCIAMENTO} Nenhuma.

\section{CONFLITO DE INTERESSE}

Os autores declararam não

haver conflitos de interesse.

$O$ estudo foi realizado no Centro

Universitário do Pará, Belém (PA), Brasil.

PALAVRAS-Chave: Educação. Ensino. Farmacêutico. Simulação de paciente.

\section{ABSTRACT}

Contextualization: Realistic simulation consists in a technique for replacing or amplifying real experiences. It is part of a new guideline perspective that enables not only technical, relational, ethical, and attitudinal skills. In the health area specifically, is presented as an attempt to reproduce the essential aspects of a clinical scenario so that when a similar scenario occurs in a real clinical context, the situation can be managed successfully. The objective of the present work is to show the experience of the realistic simulation experienced in the post-graduation of Clinical Pharmacy of the University Center of the State of Pará - CESUPA, as a teaching-learning process. Description of the experience: The research was of a descriptive and observational, with a qualitative approach. Realistic simulation as a teaching-learning tool was very well developed and applied. It prepared professionals for clinical practice, overcoming failures and fears, thus representing an important technological advance in postgraduate teaching. Final considerations: The students were able to practice the skills and competences, discuss the simulated clinical cases and this added to the construction of professional knowledge and the development of critical and clinical reasoning, both in decision making, in creating an intervention plan, and in monitoring and evaluation of results.

KEYWORDS: Education. Teaching. Pharmaceutical. Patient simulation. 


\section{Contextualização}

O processo de ensino aprendizagem vem passando por transformações ao longo dos anos, sendo que a maioria destas vem acontecendo com intuito de melhorar o contexto educacional e de formação profissional. Dentre as mudanças neste processo o avanço tecnológico se destaca, e baseado nestas transformações que o Centro Universitário do Estado do Pará - CESUPA, após a renovação da estrutura curricular, lança como proposta inovadora um curso de Pós Graduação de Farmácia Clínica e Prescrição Farmacêutica utilizando Simulação Realística.

A implementação da simulação permite aos estudantes praticar e corrigir seus erros frente a situações do cotidiano clínico, sem riscos para o paciente e com o mínimo de risco para si mesmo ${ }^{1}$. A simulação possibilita a melhora do desempenho do estudante a partir de seus próprios erros, aprendendo com as falhas para obter êxito no atendimento ao paciente, prática esta inaceitável em uma situação real ${ }^{2,3}$.

Dentre vários fatores, a formação profissional adequada é uma das responsáveis pelo sucesso de quaisquer atividades desenvolvidas na área da saúde, considerando que, em todos os níveis de atuação, será necessária formação sólida para a prática das habilidades e competências inerentes às diferentes profissões ${ }^{4-6}$.

A simulação realística compreende uma técnica para substituir ou ampliar experiências reais. $\mathrm{Na}$ área da saúde especificamente se apresenta como uma tentativa de reproduzir os aspectos essenciais de um cenário clínico para que, quando um cenário semelhante ocorrer em um contexto clínico real, a situação possa ser gerenciada pela equipe com êxito ${ }^{7}$.

O presente estudo tem como objetivo é apresentar a experiência do uso de simulação realística em uma pós-graduação em Farmácia Clinica e Prescrição Farmacêutica.

\section{Descrição da experiência}

Trata-se de um trabalho científico, do tipo relato de experiência, do curso de pós-graduação em Farmácia Clínica e prescrição farmacêutica do Centro Universitário do Estado do Pará - CESUPA, estabelecimento educacional particular de nível superior, com sede na Cidade de Belém, Estado do Pará.

As simulações realísticas foram realizadas em laboratórios equipados, os cenários eram bem realísticos, sala com mesa, maca, instrumentos específicos para ser utilizado quando necessário como esfigmomanômetro com estetoscópio e glicosímetro. Todas as simulações eram filmadas e gravadas, para posterior discussão com a turma.

Em cada módulo, eram disponibilizados casos clínicos para os grupos formados, de cada grupo era escolhido um membro para ser o farmacêutico ator. A equipe discutia o caso e criava um plano de cuidado a ser aplicado na simulação realística. As simulações envolviam pacientes (atores), os quais apresentavam problemas de saúde autolimitado, doenças crônicas, dificuldades de adesão ao tratamento farmacológico, interações medicamentosas, comorbidade, dentre outras enfermidades.

Os atores representavam pacientes de todas as classes sociais, de diferentes etnias, escolaridade, sexo e cor. E o desfecho de cada caso clínico, dependia muito, do comportamento de cada paciente (ator). Contudo, caberia ao profissional farmacêutico seguir o plano de cuidado e a tomada de decisão para aquela determinada situação. Nas simulações, uma das dificuldades encontradas pelos farmacêuticos era justamente saber conduzir e analisar qual a melhor terapia para aquele paciente.

Os professores criavam casos clínicos, nos quais os pacientes entravam em cena acompanhados. Nesses casos, surgia outra dificuldade para os farmacêuticos, que era conseguir fazer a anamnese farmacêutica. Pois os acompanhantes sempre queriam falar pelo paciente. Então, na maioria das vezes, era necessário pedir para o acompanhante sair da sala, para que o profissional pudesse ouvir o paciente e avaliar a história clinica. 
Em uma das simulações, o caso clínico era de uma farmácia de bairro, com sala para serviços farmacêuticos. A paciente (atriz) era uma paciente de 16 anos e estava com a menstruação atrasada há sete dias. Pediu à farmacêutica que lhe indicasse um medicamento para que pudesse menstruar. A farmacêutica fez a anamnese e avaliou os sinais e sintomas, indicou que ela fizesse um teste de gravidez e que comunicasse a família do que estava acontecendo.

Um hospital foi o ambiente de outro cenário da simulação realística. Esse módulo aconteceu mais da metade do curso, e foi uma das simulações mais interessante criada dentro do contexto da farmacovigilância no ambiente hospitalar. O caso clínico aconteceu, quando a equipe multiprofissional foi fazer a visita e avaliação para liberar a alta da paciente. Porém, a paciente apresentava um processo alérgico.

O médico pediu para a farmacêutica plantonista que fizesse a avaliação do que poderia ter ocasionado esse evento. A farmacêutica com olhar clínico conseguiu localizar o problema, excluindo possibilidades relacionadas a medicamentos, como interação medicamentosa e reações adversas. O processo alérgico era proveniente ao uso de hidratante corporal na noite anterior pela acompanhante. Concluindo assim o caso.

\section{Resultados e impactos}

Os cenários de simulação oferecem experiências cognitivas, psicomotoras e afetivas ${ }^{8}$. De acordo com Keskinis et al..$^{9}$ em 2016, o modelo de configuração realista poderia funcionar como uma alternativa criativa das técnicas de ensino de aulas mais tradicionais, além da simulação realística incluir técnicas que pareçam ser mais atraentes para os estudantes.

Deste modo, as experiências de ensino e aprendizagem, vividas durante todas as simulações que aconteceram nos módulos do curso foram focadas no cuidado ao paciente. As técnicas de habilidades de comunicação permitiram estreitar a relação farmacêutico-paciente, com isso, o farmacêutico consegue realizar a anamnese farmacêutica, conhecendo a história clínica do paciente. Associada a essa prática, tem-se a semiologia farmacêutica, ferramenta fundamental na simulação realística, que possibilitou uma avaliação dos sinais e sintomas, atentando para a sintomatologia apresentada pelo paciente.

De acordo com Storpirtis et al. ${ }^{10}$, a cada caso clínico simulado, o objetivo de preparar o profissional para prática clínica é alcançado. Nas simulações realísticas, após a anamnese e a semiologia, o farmacêutico foi capaz de identificar e atender as diferentes necessidades de saúde do paciente, oferecendo os serviços clínicos farmacêuticos, específico para cada um. Em muitos casos, a tomada de decisão foi um passo bastante difícil. Mas o conhecimento adquirido na prática, na discussão de cada caso clínico, somados ao pensamento crítico, foram fundamentais e embasavam a tomada de decisão.

Dependendo do caso, o farmacêutico fazia conciliação de medicamento, acompanhamento farmacoterapêutico, prescrevia terapias medicamentosas, não medicamentosas ou ambas. Fazia encaminhamento a outros profissionais, traçava plano de intervenção para acompanhar os resultados, melhorando a qualidade de vida dos pacientes. Sempre refletindo os conceitos de bases humanísticas, tendo o ser humano como um ser que dever ser valorizado, cuidado e seus valores resguardados.

Foi possível identificar dificuldades na execução dos procedimentos, além de falhas no conhecimento da farmacologia, gerando reflexão sobre a necessidade de capacitar e rever as técnicas sobre o uso do medicamento.

Deste modo, as experiências relatadas reforçam que a simulação realística é uma metodologia inovadora no processo de ensino e aprendizagem para os farmacêuticos que atuam nas farmácias comunitárias e em hospitais. 
Por meio de reprodução de cenários e situações que acontecem no cotidiano destes profissionais da saúde, verificou-se que este método de ensino favoreceu um ambiente interativo e participativo entre os participantes. A simulação realística humanizou o ensino em saúde e contribuiu para a superação das dificuldades.

\section{Considerações finais}

A simulação realística como processo de ensino-aprendizagem no ensino superior deve evoluir paralelamente às necessidades sociais. Na prática clínica, assim como em outros ramos o desenvolvimento de competências e habilidades vem sendo considerado fundamental para a formação dos profissionais. As universidades, tanto públicas quanto particulares, devem aderir à simulação realística, principalmente nos cursos da saúde. A pós-graduação em farmácia clinica, formou muitos profissionais capacitados, na prática, para cuidar e se preocupar com o paciente, contribuindo para promoção, proteção e recuperação da saúde, tanto individual quanto coletiva.

\section{Agradecimentos}

Ao Centro Universitário do Pará, pela disponibilização do espaço e mobilização dos usuários para participação no encontro.

\section{Referências}

1. McCaughey CS, Traynor MK. The role of simulation in nurse education. Nurse Educ Today. 2010;30(8):827-32. http:// dx.doi.org/10.1016/j.nedt.2010.03.005. PMid:20483188.

2. Kardong-Edgren SE, Starkweather AR, Ward LD. The integration of simulation into a clinical foundations of nursing course: student and faculty perspectives. Int J Nurs Educ Scholarsh. 2008;5(1):1-16. http://dx.doi.org/10.2202/1548-923X.1603. PMid:18673294.

3. Berragan L. Simulation: an effective pedagogical approach for nursing? Nurse Educ Today. 2011;31(7):660-3. http://dx.doi. org/10.1016/j.nedt.2011.01.019. PMid:21334797.

4. Cordeiro BC, Leite SN. O farmacêutico na atenção a saúde. Itajaí: Universidade Vale do Itajaí; 2005.

5. Collender G. The rise of interdisciplinary approaches: how scientists are working together for international development. Pharm Int. 2009;24(1):4-9.

6. Lyra D, Marques TC. As bases da dispensação racional de medicamentos para farmacêuticos. São Paulo: Pharmabooks; 2012. $270 \mathrm{p}$.

7. Vilella DS, Leite LM, Nassar MED. A simulação realística como estratégia de ensino em atendimento pré-hospitalar: um relato de experiência. São Paulo: Prefeitura de São Paulo; 2010.

8. Garbuio DC, Oliveira ARS, Kameo SY. Simulação clínica em enfermagem: relato de experiência sobre a construção de um cenário. Rev Enferm on line. 2016;10(8):3149-55.

9. Keskinis C, Bafitis V, Karailidou P, et al. The use of theatre in medical education in the emergency cases school: an appealing and widely accessible way of learning. Perspect Med Educ. 2017;6(3):199-204. http://dx.doi.org/10.1007/s40037-017-03504. PMid:28405926.

10. Storpirtis S, Nicoletti MA, Aguiar PM. Uso da simulação realística como mediadora do processo ensino-aprendizagem: relato de experiência da farmácia universitária da Universidade de São Paulo. Rev Graduação USP. 2016;1(2):49-55. http:// dx.doi.org/10.11606/issn.2525-376X.v1i2p49-55.

\section{Contribuição dos autores}

Marcilene Araújo e Valéria Araújo Barros participaram da elaboração e desenvolvimento da atividade educativa e de todas as fases de elaboração do manuscrito. Anderson Bentes de Lima participou na concepção inicial, acompanhamento, orientação do estudo e revisão crítica do manuscrito. 